\title{
MOBILIZAÇÃO DE CONHECIMENTOS NA FORMAÇÃO DO ENGENHEIRO DO AMANHÃ USANDO DESIGN THINKING
}

\author{
Miguel Angel Chincaro Bernuy - migueltrabalho@gmail.com \\ Leandro Cesardo Nascimento Bertoldi-leandrobertoldi@alunos.utfpr.edu.br \\ Joaquim Osório Brondani-joaquim.brondani@gmail.com \\ Universidade Tecnológica Federal do Paraná \\ Avenida Alberto Carazzai, 1640, Centro \\ 86300000 - Cornélio Procópio - Paraná
}

\begin{abstract}
Resumo: A construção do conhecimento durante a formação do engenheiro é o resultado de um conjunto de ações nos domínios pedagógico e de infraestrutura disponível. As propostas de reestruturação curricular, em diversos cursos de engenharia, têm desafios que percorrem estes domínios. A materialização do macroplanejamento, cuja representação está no projeto pedagógico do curso, está na elaboração e execução de planos de ensino das disciplinas. Neste trabalho, é apresentado detalhadamente a estrutura e a execução da parte de uma disciplina na área de microcontroladores, que corresponde a mobilização de conhecimentos específicos da área. Ou seja, será apresentada a parte que corresponde a integração dos conhecimentos, a avaliação processual da disciplina e o desenvolvimento de competências comportamentais do engenheiro do amanhã (inovador e responsável). A disciplina usada é Sistemas Microcontrolados e o método utilizado na estruturação desta parte da disciplina é o Design Thinking. Os resultados podem ser observados em duas operações: divergente e convergente, indicando a natureza de processos criativos e complexos associados a conceitos e conteúdos relativos à área específica da disciplina.
\end{abstract}

Palavras-chave: Design Thinking. Mobilização de conhecimentos. Sistemas Microcontrolados.

\section{INTRODUÇÃO}

$\mathrm{Na}$ formação de um engenheiro, saber como coletar e analisar informações torna-se imprescindível a este profissional, dado que no exercer de sua função terá que lidar com o desenvolvimento de soluções a partir de problemas muitas vezes associados a tarefas do cotidiano social. Entender a problemática, idealizar uma solução, prototipar e validar são fases essenciais para a construção da resolução de problemas.

Durante um curso de engenharia, são apresentadas disciplinas de formação profissional que trabalham, muitas vezes em equipes, com a elaboração de soluções para problemas. O Design Thinking, cuja origem vem do campo do design, sistematiza a busca de soluções inovadoras, focado em necessidades reais de mercado.

Disciplinas como Sistemas Microcontrolados, apresentam uma carga teórica, porém, permitem também ao aluno aplicar estes conhecimentos em resoluções de problemas reais, os quais é possível a utilização da abordagem do Design Thinking, como também o desenvolvimento de habilidades organizacionais envolvendo divisão de tarefas, membros responsáveis e cronogramas um processo muito bem visto no mercado de trabalho. 
Visando os processos que levam a formação de um engenheiro, este trabalho apresenta todas as etapas utilizadas por uma equipe de estudantes na disciplina de Sistemas Microcontrolados, englobando desde a definição de uma problemática até a prototipagem e validação da solução.

\section{METODOLOGIA}

A metodologia utilizada para a elaboração deste trabalho baseia-se no Design Thinking, no qual trata-se de um modelo que se manifesta em etapas de imersão, ideação e prototipação, e também durante a implantação, até tornar o projeto uma realidade.

Esta abordagem, apresenta um modelo mental que alterna divergência e convergência. Quando atua o modo divergente, busca-se criar opções para a resolução de problemas, já quando atua no modo convergente, é o momento de fazer escolhas, ou seja, priorizar a opção que mais se adequa dentre as outras. (MELO e ABELHEIRA, 2015).

Seguindo os princípios de Design Thinking, onde o mesmo pode ser compreendido como um conjunto de etapas, tais como Descoberta, Interpretação, Ideação, Implementação e Evolução, organizada pela IDEO (IDEO, 2012), este trabalho foi desenvolvido. Sendo dividido cada etapa durante o decorrer da disciplina de Sistemas Microcontrolados, como é descrito neste trabalho.

\section{DEFINIÇÃO DA PROBLEMÁTICA}

No início realizou-se o levantamento de problemáticas de diferentes áreas ou cenários, podendo ser problemas do setor industrial, comercial ou mesmo do cotidiano social, para estes seriam descritos de acordo com três perguntas: "Por quê?", "O quê? " e "Para quem?".

\subsection{Resumo das problemáticas}

Gerenciamento de Lavanderia Compartilhada: Com uma grande adesão a esse serviço podem ocorrer problemas de organização, principalmente na questão de agendamento para uso das máquinas. Os métodos de agendamento que normalmente são utilizados são por meio de cadernetas, quadros ou mesmo apenas por comunicação verbal, e isso acarreta alguns pequenos inconvenientes que podem ser frustrantes.

Janelas Abertas em Períodos de Chuva: Muitas das pessoas esquecem de fechar a janela ao sair de casa e justamente quando isso acontece, começa a chover, o que nos deixa frustrados, além de ocasionar muitos problemas.

Controlar Temperatura do Chuveiro por Aplicativo: Devido às variações climáticas, é necessário que estejamos sempre ajustando a chave seletora do chuveiro para que a água fique em uma temperatura agradável.

Sensor na Torneira do Bebedouro da Universidade: Por diversas vezes já foi observado que ao utilizar o bebedouro para encher um recipiente (garrafa ou copo), há um desperdício de água entre o momento que o usuário percebe que está cheio e realize o fechamento da torneira.

Alarme de Carro Controlado por Aplicativo: Um dos problemas que podem ser solucionados é a questão de furto de automóveis, pois somente o aviso sonoro pode não ser tão eficaz, dado a distância ou deficiências auditivas do proprietário.

Controle de Temperatura em Estufas: as estufas precisam manter a temperatura em um certo intervalo para que não afete a plantação, e alguns problemas de controle e regulagem dessa temperatura ocorrem por avarias externas diminuindo a eficiência energética do equipamento. 
Critérios para seleção da problemática

Dado o levantamento das problemáticas pelos membros da equipe, foram propostos critérios de seleção, visando filtrar o problema mais relevante:

[1] Viabilidade: Obedece às características tecnológicas passíveis de implementação?

[2] Usabilidade: Facilidade com que as pessoas possam empregar a ferramenta.

[3] Implementação: Tempo para implementação, é possível dentre o disponibilizado na disciplina.

[4] Requisitos: Os materiais que compõem a implementação da aplicação são de fácil acesso?

No final, atribui-se uma nota de 0 a 5, com 0 representando "Não satisfaz" e 5 "Satisfaz Completamente", classificando-as por meio da soma de cada nível determinado nos critérios, como mostra o Quadro 1.

\begin{tabular}{|c|c|c|c|c|c|c|}
\hline \multicolumn{2}{|r|}{ Problemas } & Viabilidade & Usabilidade & Implementação & Requisitos & Total \\
\hline [1] & Lavanderia & 5 & 5 & 4 & 4 & 18 \\
\hline [2] & Janelas Abertas & 5 & 2 & 2 & 2 & 11 \\
\hline [3] & Temperatura Chuveiro & 4 & 3 & 3 & 3 & 13 \\
\hline [4] & Sensor Bebedouro & 4 & 5 & 3 & 3 & 15 \\
\hline [5] & Alarme Aplicativo & 3 & 4 & 2 & 2 & 11 \\
\hline [6] & Temperatura Estufas & 4 & 5 & 3 & 2 & 14 \\
\hline
\end{tabular}

Fonte: Autoria própria.

Com o resultado da soma, a problemática escolhida foi o Gerenciamento de Lavanderia Compartilhada, pois teve maior número de pontuação nos critérios estabelecidos e devido a alta demanda em certos condomínios que possuem lavanderia compartilhada, pois existe a ocorrência de conflitos decorrentes da má organização dos horários de agendamento, entre outros fatores.

\subsection{Pesquisa de mercado}

Realizou-se uma pesquisa para ter uma quantidade de pessoas que já utilizam o serviço de lavanderia compartilhada, se possui alguma forma de agendamento de horários, e se ocorrem problemas relacionado a este serviço. Para obter tais informações foi elaborado um survey, que permite dois fluxos de respostas "Figura 1", permitindo que dois grupos de perguntas, sendo Perguntas A, pessoas que já utilizaram lavanderias compartilhadas onde é apresentado perguntas mais específicas sobre o tema, e Perguntas B, pessoas que vieram a responder o questionário, porém não utilizaram lavanderias compartilhadas, para estas apresentamos uma descrição da problemática e questionamos a respeito da elaboração de uma possível solução. 
Figura 1 - Fluxo de Questões aplicados no survey.

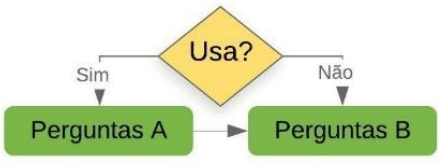

Fonte: Autoria própria.

Já utilizou lavanderia ou máquina de lavar roupas compartilhada?

\section{Perguntas A}

Havia algum tipo de agendamentoO agendamento para a utilização da máquina é visível a todos? E como é feito o agendamento?

Recebeu instruções sobre o funcionamento da lavanderia? Como exemplo, horários permitidos, quantas vezes pode lavar por semana, etc.

Já aconteceu de esquecerem roupa na máquina e atrapalhou o horário de outro?

Já agendou o horário e mesmo assim outra pessoa começou a utilizar a máquina?

Já ocorreu agendamentos excessivos para utilização da máquina por somente uma pessoa?

Já ocorreu impasses entre alguns moradores, diretamente ou indiretamente, por causa do uso da máquina de lavar?

\section{Perguntas $B$}

O que acharia de poder agendar o horário por meio de um aplicativo?

O que acharia de um sistema de controle de acesso por cards ou bottons, impedindo o acesso por quem não aguentou previamente.

\subsection{Resultados da Pesquisa}

O questionário foi elaborado utilizando a plataforma Google forms e divulgado no grupo da UTFPR, Universidade Tecnológica Federal do Paraná campus de Cornélio Procópio, em uma rede social, apresentando um total de 69 respostas.

Analisando o resultado do levantamento, constatou-se que mais da metade das pessoas que responderam o questionário utilizam máquina de lavar compartilhada "Figura 2" seguindo o fluxo de Perguntas A aproximadamente 63\% "Figura 3" não possuíam nenhuma forma de agendamento, tendo 58,3\% impasses entre alguns moradores "Figura 4", direta ou indiretamente, por causa do uso da máquina de lavar, ou seja, pode-se evidenciar uma real problemática envolvida nesta tarefa cotidiana, por meio de ambos os fluxos pode-se observar também que 55,9\% das pessoas achariam ótimo um sistema de organização e controle de acesso às máquinas de lavar, impedindo o acesso por quem não agendou previamente. 


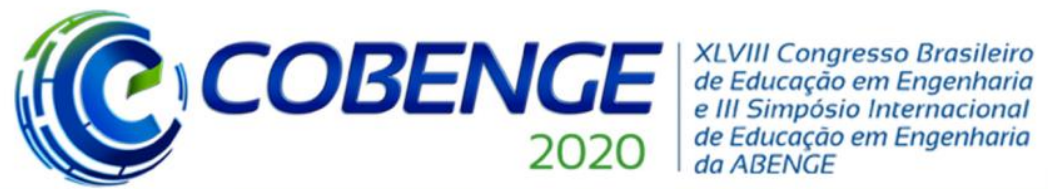

"Os desafios para formar hoje o engenheiro do amanhã"
Figura 2 - Gráfico A.

Já utilizou lavanderia ou máquina de lavar roupas compartilhada?

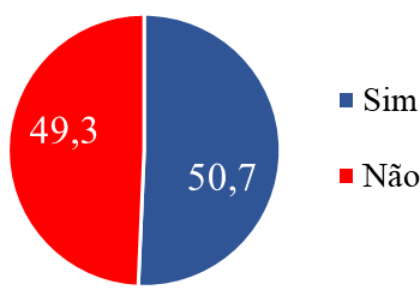

Fonte: Autoria própria.
Figura 3 - Gráfico B.

Havia algum tipo de agendamento?

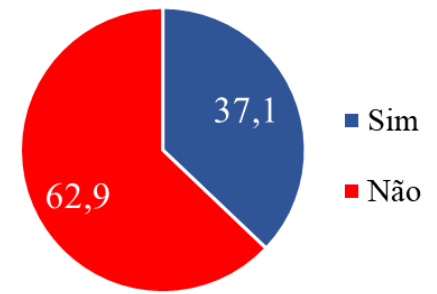

Fonte: Autoria própria.

Figura 4 - Gráfico C.

Já ocorreram impasses entre alguns moradores, diretamente ou indiretamente por causa do uso da máquina de lavar?

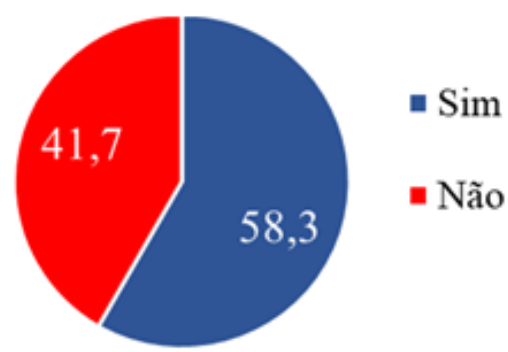

Fonte: Autoria própria.

\section{PROTOTIPAGEM E VALIDAÇÃO}

\subsection{Ferramenta de Organização Trello}

Para a organização das atividades foi utilizado a plataforma Trello, sendo uma ferramenta de colaboração que organiza seus projetos em quadros, onde cada tarefa pode ser dividida em cartões, e estes podem ser atribuídos ao responsável pela ação, uma descrição e data de entrega da mesma.

Com o auxílio dessa ferramenta, montou-se um quadro seguindo o formato de Kanban, um sistema ágil e visual para controle de produção ou de gestão de tarefas (ESPINHA, 2019), possuindo sinalização que controla os fluxos de produção, permitindo que uma ação seja trocada por outro sistema de sinalização, tais como Tarefas, Para Fazer, Fazendo e Feito, tornando possível a otimização, acompanhamento, distribuição e organização das tarefas no processo de desenvolvimento.

\subsection{Ideação e Implementação}

Após a pesquisa realizada, e análise dos dados obtidos, elaboramos o levantamento de requisitos do problema, de tal forma a possibilitar elaborar uma solução baseada em uma melhor organização do uso do espaço coletivo, esta seria a criação de um aplicativo para realização do agendamento, a qual atingiria a problemática da visualização, sincronização e organização dos horários entre os usuários da máquina, em conjunto com um hardware de controle de acesso, garantindo a reserva de um horário. Através de uma conexão via Bluetooth, que permite comunicação com o dispositivo, somente aquele que está agendado para um determinado período conseguirá utilizar a máquina de lavar roupas. 
Assim, dado o levantamento dos requisitos do problema, partiu-se então para as especificações da solução, sendo dividida em duas partes, hardware de controle de acesso e o aplicativo móvel.

\section{Levantamento de requisitos de hardware e software}

Para a implementação de um protótipo do hardware, responsável pelo controle de acesso, foi utilizado os seguintes componentes: Microcontrolador Arduíno UNO, Módulo Bluetooth RS232 HC-05, Relé de Estado Sólido SSR-25 e Lâmpada Incandescente.

A plataforma MIT App Inventor foi utilizada para o de desenvolvimento do aplicativo, sendo um ambiente de programação visual intuitivo que permite criar aplicativos totalmente funcionais para smartphones e tablets. A ferramenta é baseada em blocos, o que facilita a criação de aplicativos complexos e de alto impacto em um tempo significativamente menor que os ambientes de programação tradicionais, contudo o desenvolvimento do app se torna mais simples e otimizado.

Desenvolveu-se uma diagrama de funcionamento da aplicação "Figura 5", considerando a jornada de interação do usuário até a máquina de lavar, ou seja, por meio do aplicativo, ele realiza seu cadastro somente no primeiro uso, e por seguinte realiza o agendamento em um horários disponíveis, dado a sincronização das reservas no banco de dados, com um agendamento realizado, também por meio do aplicativo, o usuário realizar uma conexão com o hardware utilizando comunicação Bluetooth, e libera o acesso à máquina de lavar, se satisfazer as condições de data e hora preestabelecidas no agendamento.

Já definido a plataforma de desenvolvimento do aplicativo, elaborou-se o layout da aplicação "Figura 6", e posteriormente construiu a programação por meio dos blocos, seguindo a regra de funcionamento da aplicação. Para a sincronização entre os aplicativos foi utilizado um banco de dados não relacional, sendo o Firebase um serviço de cloud para aplicações web e mobile do Google.

Figura 5 - Diagrama de fluxo para funcionamento da aplicação.

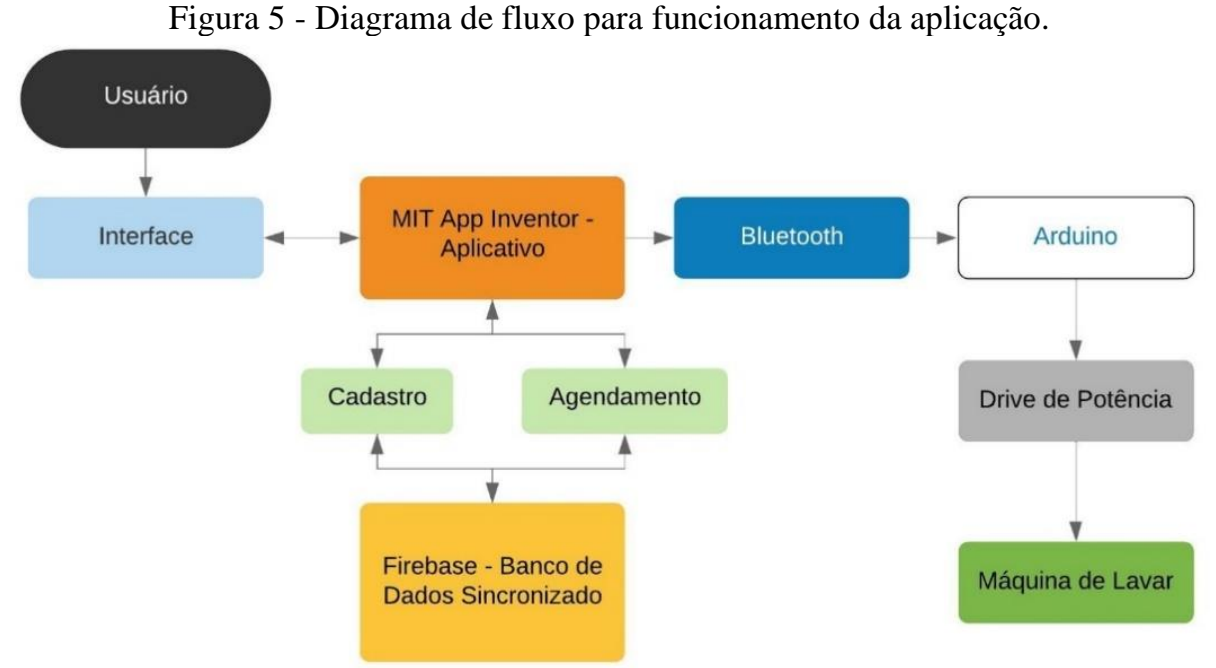

Fonte: Autoria própria. 


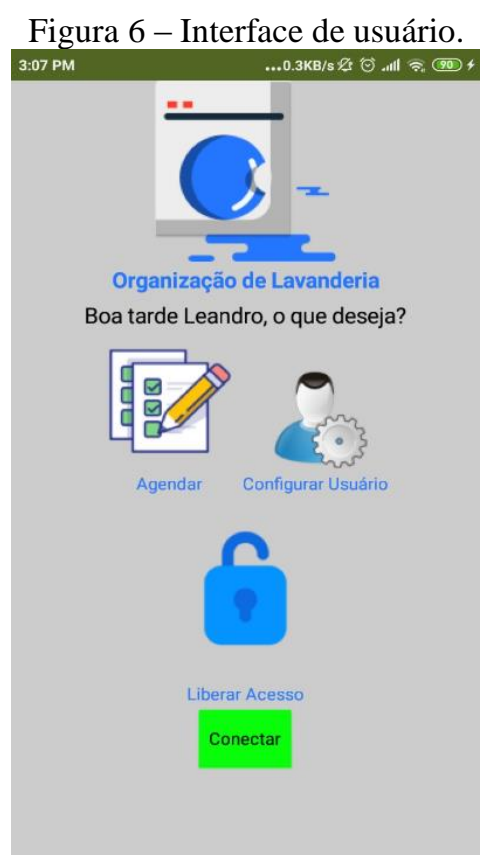

Fonte: Autoria própria.

Na elaboração do circuito do dispositivo de controle de acesso, foi utilizado o Arduino Uno que possui um próprio ambiente de desenvolvimento que implementa a Linguagem $C$. Sendo a peça central para o desenvolvimento deste projeto, o Arduino é responsável por fazer a comunicação com o aplicativo através do Módulo Bluetooth HC-05 e também enviar um sinal de tensão para o Relé de Estado Sólido, que neste caso atua como chave e, quando recebe este sinal, fecha o contato e permite que a máquina de lavar entre em funcionamento (FOTEK, 2020). Para realização de teste em laboratório foi utilizada uma lâmpada ligada à rede elétrica de $127 \mathrm{~V}$ simulando a máquina de lavar.

\subsection{Pesquisa de feedback pós Prototipação}

Foi realizado uma segunda pesquisa para saber o que possíveis usuários opinam sobre a solução encontrada, sendo mostrado um vídeo (https://youtu.be/9u2hoehnheq) de como ficou o uso do aplicativo em conjunto com o hardware que garante o acesso a máquina. As cinco perguntas a seguir estavam no questionário:

Você acha que esta solução ajudaria a melhorar os horários de agendamento das máquinas?

Qual a possibilidade de você indicar essa solução para alguém que tenha esse problema? (Escala de 0 a 5, onde 0 quer dizer nenhuma possibilidade e 5 alta possibilidade)

Se interessaria em utilizar uma solução baseada nesse protótipo?

Tem alguma sugestão para complementar ou melhorar esta solução?

Qual valor você estaria disposto a pagar?

Nas Figuras 8, 9 e 10 estão apresentadas algumas das respostas para essas perguntas. 
Figura 7 - Gráfico D.

Se interessaria em utilizar uma solução baseada nesse protótipo?

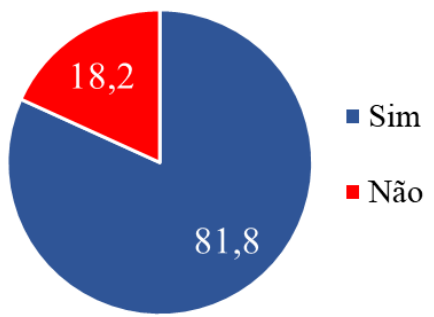

Fonte: Autoria própria.
Figura 8 - Gráfico E.

Você acha que esta solução ajudaria a melhorar os horários de agendamento das máquinas?

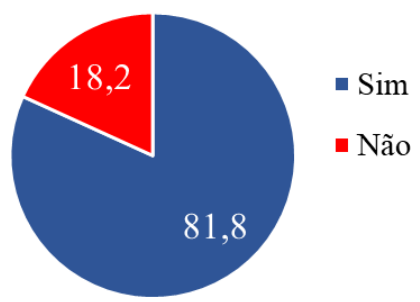

Fonte: Autoria própria.

Figura 9 - Gráfico F.

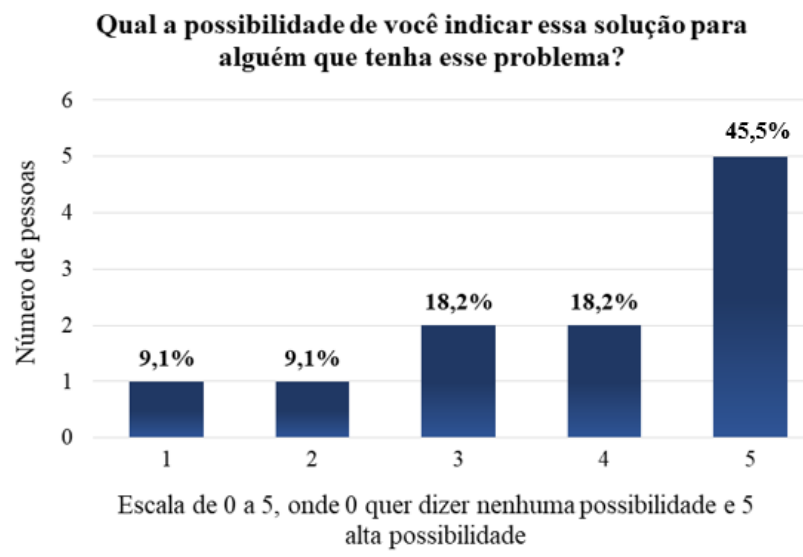

Fonte: Autoria própria.

\section{CONSIDERAÇÕES FINAIS}

O trabalho mostra de maneira detalhada uma abordagem de mobilização de conhecimentos da disciplina Sistemas Microcontrolados, permitindo o desenvolvimento de competências atitudinais de maneira articulada com competências técnicas, muito importantes para formação do engenheiro do amanhã.

Com o resultado da pesquisa final constatou-se que o projeto de uma solução para lavanderias compartilhadas foi bem aceito pelas pessoas, visto que $81,8 \%$ dos entrevistados responderam que o aplicativo proposto ajudaria a melhorar os horários de agendamento das máquinas e na mesma proporção se interessariam em utilizar uma solução baseada no protótipo sugerido. Igualmente, sobre a possibilidade de indicar a solução para outras pessoas, todos responderam que provavelmente indicariam, sendo 45,5\% com alta possibilidade de indicar a solução para outros usuários.

Vale acrescentar, que a ferramenta proposta é viável, de fácil manuseio e grande utilidade, levando em consideração que o desenvolvimento de um protótipo inicial é passivo de futuras modificações e ajustes. Visto que, importantes aspectos do desenvolvimento de produtos são harmonizados com a construção da solução apresentada.

Desta forma, é demonstrada uma maneira diferente de abordagem do conteúdo de sala de aula aplicado no cotidiano da comunidade externa. Portanto, trabalhou-se com um dos grandes desafios atuais para os estudantes dos cursos de engenharia, que procuram na sua formação a experiência de como será aplicado o conhecimento teórico na prática depois que se formarem. 


\title{
Agradecimentos
}

Agradecemos à UTFPR que disponibilizou a utilização da infraestrutura acadêmica para que fosse possível o desenvolvimento deste trabalho em meio a disciplina de Sistemas Microcontrolados ministrada nos cursos de Engenharia de Computação e Engenharia Elétrica. Agradecemos ao discente Johnny de Oliveira Camargo que participou no desenvolvimento de ações relacionadas a este projeto.

\section{REFERÊNCIAS}

ESPINHA, Roberto Gil. Kanban: O que é e TUDO sobre como gerenciar fluxos de trabalho. Disponível em: https://artia.com/kanban/. Acesso em: 27 mai. 2020.

FOTEK. Datasheet fotek ssr. Disponível em: https://img.filipeflop.com/files/download/Datasheet_fotek_ssr.pdf. Acesso em: 22 mai. 2020.

IDEO. Design Thinking for Educators. 2th. ed. New York: IDEO, 2012.

MELO, Adriana; ABELHEIRA, Ricardo. Design Thinking \& Thinking Design: Metodologia, ferramentas e uma reflexão sobre o tema. São Paulo: Editora Novatec. 2015.

\section{KNOWLEDGE MOBILIZATION IN THE TRAINING OF TOMORROW ENGINEER USING DESIGN THINKING}

\begin{abstract}
The construction of knowledge during the training of the engineer is the result of a set of actions in the domains of pedagogy and available infrastructure. The curricular restructuring proposals, in several engineering courses, have challenges that run through these domains. The materialization of macro planning, whose representation is in the pedagogical project of the course, is in the preparation and execution of teaching plans for the subjects. In this work, the structure and execution of part of a discipline in the microcontroller area is presented in detail, which corresponds to the mobilization of specific knowledge in the area. In other words, the part that corresponds to the integration of knowledge, the procedural assessment of the discipline and the development of behavioral skills of the engineer of tomorrow (innovative and responsible) will be presented. The discipline used in Microcontroller Systems and the method used in structuring this part of the discipline is Design Thinking. The results can be observed in two operations: divergent and convergent, indicating the nature of creative and complex processes associated with concepts and contents related to the specific area of the discipline.
\end{abstract}

Keywords: Design Thinking. Knowledge Mobilization. Microcontroller Systems. 